\title{
Mental Health in the Times of Corona: A model for Positive Mental Health During the Global Pandemic
}

\author{
Jain Mathew ${ }^{1} \cdot$ Roseline Florence Gomes $^{2} \cdot$ Sheeba Bhaskar $^{1} \cdot$ Preksha Yadav $^{1,3}$. \\ Sridevi Nair ${ }^{1}$ (i)
}

Received: 4 June 2020/ Accepted: 1 June 2021 / Published online: 12 July 2021

(C) National Academy of Psychology (NAOP) India 2021

\begin{abstract}
As the world comes to terms with the changes brought on by the appearance and spread of Covid-19, there is an increase in fear and anxiety levels. The World Health Organization has stressed on the need to focus on mental health, as the epidemic affects both young and old. One of the biggest challenges is that the situation has no precedent. This has led to the creation of uncertainty and doubt. The current study focusses on studying the positive mental health of the Indian population. For the study, the researchers have restricted the scope to include only Generation $Y$ and Generation $Z$, as they form a majority of the working population and literature suggests that negative moods, stressors and apprehensions are far more prevalent among the younger sections of the society. The findings suggest that positive mental health is not dependent on gender or age. However, some of the dimensions do show significant variance. The researchers have also proposed a model of Positive Mental Health based on the dimensions of the Positive Mental Health Instrument.
\end{abstract}

Keywords Positive mental health · Pandemic · Covid-19 . Age $\cdot$ Gender

\section{JEL Classification 131}

Sridevi Nair

sridevi.nair@res.christuniversity.in

1 School of Business and Management, CHRIST (Deemed To Be University), Bangalore, India

2 Department of Psychology, CHRIST (Deemed To Be University), Bangalore, India

3 CMR Institute of Technology, Bangalore, India

\section{Introduction}

The Indian environment is undergoing a plethora of changes in the current pandemic state of lockdown, and these variations signify a gradual shift in mindsets. The results of these modifications in terms of affective, interpersonal, spiritual and coping strategies; are the unpleasant outcomes brought about by Covid-19 (Kazmi et al., 2020).

The Indian lockdown was implemented on 24th March 2020 by the Prime Minister. The lockdown has further led to affective distress, fears of exhibiting social skills and coping mechanisms in the society (Kazmi et al., 2020). Zhang et al., (2020) proposed that the pandemic state developed psychological divisions among the general public and questioned the familial support systems ability to guide youth and young adults'through this transitional phase of health emergency. The pandemic has also brought into consideration the mental dysfunctions across cultures and geographic spaces. According to a recent report drafted by John Hopkins University, over 3,606,038 individuals were battling with health change due to Covid-19, and 252,151 deaths have taken place across the globe (UrbinaGarcia, 2020). The global pandemic state has created an emergent need for the policy-makers to focus on the favorable mental health. It has also brought into focus the lack of studies that focus on the psychological health of individuals, especially based in similar unpredictable contexts, as the pandemic (Wang et al., 2020). Thus, the epidemic alert has also revived the need for context-specific mental health depictions among generations (Urbina-Garcia, 2020). Therefore, the current study was undertaken to explore the positive mental health of the active members of the study. 


\section{Literature Review}

The outbreak of a pandemic has the potential to impact not only existing illnesses but also to cause distress among caretakers, affected persons and the general population; thereby leading to an increase in mental symptoms. The emergence of COVID-19 has led to growing confusion anxiety and fear (WHO, 2020). The mental health of the Indian population has been fluctuating during the pandemic. Modifications in interpersonal skills, such as physical distancing have added to perceptions of lack of affective support from one's environment (Kazmi et al., 2020). However, there appears to exist a lack of evidencebased researchers that evaluates favorable or positive mental health of people (Elmer et al., 2020). The current study aims to fill this gap by exploring the dimensions of favorable mental health across age groups and gender.

There have been innovations in the functioning of dynamic models that incorporated positive mental health among different age groups and sketched the attributes of psychological well-being. This section describes some of the key theories, models and literature in the area of Positive Mental Health. One of the key models in the area is the self-determination model that defined motivational skills as adapting to a positive growth mindset with the presence of turbulence within the environment (Sheldon, 2012). This theory focusses on the innate tendencies of individuals to apply skills of independence and interpersonal qualities to promote positive mental and satisfaction; and proposes a holistic affect.

Fredrickson (2004) crafted a visionary perspective of widening one's personalized ability to develop novel cognitive skills or thinking capacities, that encourage general coping and improves social connectivity. This theoretical framework governed the functioning of global emotions, drastically changed the perception of mental health. It proposed that people have an innate tendency of compartmentalizing mental health dimensions through affect and integrating its components into realistic settings.

Seligman (2011) formulated the well-being model of positive mental health that revolved around the dimensions of positive affect, engagement, relationships, meaning in life and accomplishment; across dynamic ages irrespective of gender. According to the theory, the key is to develop skills for identifying positive emotions and expanding interactions within the community, to reinforce meaningful relationships, coping tendencies, spirituality and holistic emotional stability. These factors interact in a global unit that strengthens positive mental health.

Vaigankar et al. (2011) transformed the conceptualization and understanding of mental health. Mental health was traditionally researched among the western population and did not include interpersonal skills, affect, spirituality and coping methods, that define the holistic emotional reactions of people. Vaigankar et al. (2011) were critical in making positive mental health-relevant and important for realizing and strengthening of potential for building coping skills in times of crisis and stressful events. They believed that this would encourage individuals to participate and develop positively in the community, through the realization of personalied positive psychological constructs. Kazmi et al. (2020) stated that the emergent pandemic state has sketched the global need to sensitize people and found that negative moods, stressors and apprehensions were far more prevalent among youth and young adults. This has led to interruptions in interpersonal qualities with regards to building a network and has transformed the affective mental health in people (Elmer et al., 2020). It has also necessitated the understanding of the emotional, social and personal capacities of individuals in the era of social distancing. Spirituality, corresponding to mental health, across diverse age groups and cultures are less explored and has been linked to overtly religious actions in stressful times (Ganga \& Kutty, 2013). Therefore, the current study is undertaken to strategize knowledge of positive mental health across youth and young adults; in building awareness about the mental health states in the pandemic context. Unavailability of studies in understanding dimensions of social skills, emotional support, spirituality, coping and global affect among individuals suggest a need for the current study.

\section{Materials and Methods}

The study proposed to analyse the positive mental health state during the lockdown. The objectives of the research were as follows;

- To analyse the variance of dimensions of positive health across genders

- To analyse the variance of dimensions of positive health across age groups.

- To propose a model of positive mental health

\section{Participants}

The sample for the study comprised of male and female members of Generation $Y$ and Generation $Z$. Generation $Z$ was represented by individuals in the age group of $18-25$, while Generation $Y$ included individuals in the age group of 26-40. These generations were chosen because they make up a bulk of the working population in India and will form a bulk of the active population in the coming years (OECD, 2020). In addition, Kazmi et al. (2020) found that negative 
moods, stressors and apprehensions were far more prevalent among younger sections of the population. The members of the generation from 26 to 40 years, have also been found to increasingly battle with thoughts of narcissism and personal inadequacies; which in turn affect their mental functioning (Ellin, 2014). Similarly, Generation $Z$ is said to suffer from psychological upheavals due to variations in ego, driven by changes in technological innovations and differences in caregiver support (Leskauskas, 2020).

\section{Procedure}

The researchers shared a link to the survey on Google Forms, with the potential respondents. The respondents were first provided with a brief description of the study. The next step required that the participants indicate their consent to participate in the study. On receiving their informed consent, the participants were asked questions regarding their age and country of residence. This was done to ensure that the participants belonged to the target population. Only participants belonging to the target age groups and residing in India were directed to the survey and allowed to record their responses.

The collected data were cleaned and coded in Excel. After the initial processing of the data, which included the removal of outliers, the researchers had a sample of 197 usable datasets. The final data were analyzed using SPSS. The results of the analysis have been presented in the next section. The first section of the analysis involved the analysis of the variables viz-a-viz age and gender. For the correlation, regression and mediation analysis, the researcher chose to analyse the complete data. This was done to ensure that the sample size was acceptable for the required analysis. The formula used to calculate the required sample size was proposed by Green (1991). He suggested that for analysis of relationships;

$N>104+m$

where, $N=$ sample size; $M=$ no. of predictors or Independent Variables.

\section{Measures}

The dimensions of positive mental health were borrowed from Vaigankar et al. (2011). Since the Positive Mental Health Instrument was created and validated in the Asian Population, the researchers felt it would be better suited to the Indian population as compared to instruments created and validated in the West. The dimensions identified and included in the study are Emotional Support $(E)$, Interpersonal Skills (I), Spirituality (S), General Coping (GC) and Global Affect $(G)$. The items to measure each variable were adapted from the Positive Mental Health instrument. The original statements were slightly modified to stress on the context of the lockdown. For example, while the original statement read "When I feel stressed....", the modified statement was "During the current lockdown, when stressed...". All items were measured on a 5-point scale. The modifications were validated by a group of experts.

\section{Results}

The first step in the analysis involved analyzing the reliability of the scales that were used for the analysis. All the scales have a Cronbach $\alpha$ value greater than the accepted value of 0.7 . Table 1 provides the reliability scores for the scales.

The next step was the analysis of variance across the gender categories. Table 2 provides the mean scores of the variables, across the gender categories. For the current sample, an analysis of the mean scores of the variables suggests that the score for Females was higher than that for Males. In other words, on all the variables, Female respondents appeared to have marked themselves higher than the male respondents. A test for significance of the variance was carried out using a $t$-test. The results of the $\mathrm{t}$-test have been provided in Table 2 .

The results of the t-test suggest that the difference in mean values is only significant for the variable Spirituality. An item wise analysis suggested Females were likely to mark themselves higher on all statements except for the statement "My religious beliefs influence the way I live". On this item, males had a higher mean score than Females.

The analysis of the mean values of the variables across age groups has been provided in Table 3. The members of Generation $Y$ had higher mean scores for all variables. This suggests that Generation $Y$ respondents perceive themselves to be more positive than the Generation $Z$ respondents. The significance of this difference was analyzed using a $t$-test. The results of the $t$-test have been provided in Table 3. The results of the $t$-test suggest that the difference in mean values is only significant for the variable Interpersonal Skills.

The researchers then conducted a correlation analysis to understand the relationship between the independent variables of General Coping, Emotional Support, Spirituality and Interpersonal Skills and the dependent variable of Global Affect. The result of the correlation analysis has been provided in Table 4.

All variables were found to be significantly correlated with $p<0.01$, except for Spirituality. Emotional support was found to have the strongest relation with Global Affect $(r=0.331)$. 
Table 1 Reliability scores

\begin{tabular}{lll}
\hline & Variable & $\begin{array}{l}\text { Cronbach } \\
\alpha\end{array}$ \\
\hline 1 & General coping & 0.886 \\
2 & Emotional support & 0.892 \\
3 & Spirituality & 0.919 \\
4 & Interpersonal & 0.921 \\
5 & skills & \\
\hline
\end{tabular}

Table 2 Variance vis-a-vis gender of respondents

\begin{tabular}{llrllll}
\hline & $N$ & Mean & $t$ & Df & Sig & \\
\hline GC & Male & 44 & 3.5227 & -1.807 & 195 & 0.072 \\
& Female & 153 & 3.7712 & & & \\
$E$ & Male & 44 & 3.5227 & -1.48 & 195 & 0.141 \\
& Female & 153 & 3.7712 & & & \\
$S$ & Male & 44 & 3.0455 & -3.27 & 195 & 0.001 \\
& Female & 153 & 3.6732 & & & \\
$I$ & Male & 44 & 3.9318 & 0.733 & 195 & 0.464 \\
& Female & 153 & 3.8301 & & & \\
$G$ & Male & 44 & 3.7727 & -1.491 & 195 & 0.138 \\
& Female & 153 & 4.0196 & & &
\end{tabular}

Table 3 variance vis-a-vis age of Respondents

\begin{tabular}{llrllll}
\hline & Age & \multicolumn{1}{c}{$N$} & Mean & $t$ & df & Sig \\
\hline GC & $18-25$ & 103 & 3.699 & -0.303 & 195 & 0.762 \\
& $26-40$ & 94 & 3.734 & & & \\
$E$ & $18-25$ & 103 & 3.6408 & -1.119 & 195 & 0.264 \\
& $26-40$ & 94 & 3.7979 & & & \\
$S$ & $18-25$ & 103 & 3.4466 & -1.105 & 195 & 0.271 \\
& $26-40$ & 94 & 3.6277 & & & \\
$I$ & $18-25$ & 103 & 3.7379 & -2.102 & 195 & 0.037 \\
& $26-40$ & 94 & 3.9787 & & & \\
$G$ & $18-25$ & 103 & 3.9515 & -0.196 & 195 & 0.844 \\
& $26-40$ & 94 & 3.9787 & & & \\
\hline
\end{tabular}

Among the independent variables, the strongest relationship was found to be between Spirituality and Emotional Support $(r=0.531)$.

The researchers then attempted to build a predictive model. The initial regression analysis suggested that only Emotional Support was significant in predicting Global Affect. The summary of the regression analysis has been provided in Table 5.
Table 4 Correlation matrix

\begin{tabular}{|c|c|c|c|c|c|c|}
\hline & GC & E & $S$ & $I$ & $G$ & \\
\hline \multirow[t]{2}{*}{ GC } & $\begin{array}{l}\text { Pearson } \\
\text { Correlation }\end{array}$ & 1 & & & & \\
\hline & Sig. (2-tailed) & & & & & \\
\hline \multirow[t]{2}{*}{$E$} & $\begin{array}{l}\text { Pearson } \\
\text { Correlation }\end{array}$ & $.475^{* *}$ & 1 & & & \\
\hline & Sig. (2-tailed) & .000 & & & & \\
\hline \multirow[t]{2}{*}{$S$} & $\begin{array}{l}\text { Pearson } \\
\quad \text { Correlation }\end{array}$ & $.268^{* *}$ & $.531^{* *}$ & 1 & & \\
\hline & Sig. (2-tailed) & .000 & .000 & & & \\
\hline \multirow[t]{2}{*}{$I$} & $\begin{array}{l}\text { Pearson } \\
\text { Correlation }\end{array}$ & $.372^{* *}$ & $.427^{* *}$ & $.304^{* *}$ & 1 & \\
\hline & Sig. (2-tailed) & .000 & .000 & .000 & & \\
\hline \multirow[t]{2}{*}{$G$} & $\begin{array}{l}\text { Pearson } \\
\text { Correlation }\end{array}$ & $.286^{* *}$ & $.331^{* *}$ & .099 & $.279^{* * *}$ & 1 \\
\hline & Sig. (2-tailed) & .000 & .000 & .165 & .000 & \\
\hline
\end{tabular}

Given the strong relationship between Emotional Support and the other variables, the researchers then analyzed for the possibility of Emotional Support mediating the impact of General Coping, Interpersonal Relations and Spirituality on Global Affects. Mediation analysis was carried out using the Process macros in SPSS. The results have been presented in Table 6 .

The results of the analysis suggest that Emotional support partially mediated the relationship between General Coping and Global Affect. It was also found that Emotional support partially mediated the relationship between Interpersonal Relations and Global Affects. However, Emotional Support was found to fully mediate the relationship between Spirituality and Global Affect. Thus, the relationship between General Coping and Global Affect and Interpersonal Relations and Global Affect was further strengthened by the mediating effect of Emotional Support. While the relationship between Spiritual Intelligence and Global Affect was only significant.

Based on the analysis, the researchers propose the following model (Fig. 1) of relationships between the variables;

Table 5 Summary of Regression Analysis

\begin{tabular}{lllllll}
\hline Model & $R$ & $R$ square & $\begin{array}{l}\text { Adjusted } \\
R \text { square }\end{array}$ & $\begin{array}{l}\text { Std. error of the } \\
\text { estimate }\end{array}$ & Sig \\
\hline 1 & $.382^{\mathrm{a}}$ & 0.146 & 0.133 & 0.90402 & 11.021 & $.000^{\mathrm{b}}$
\end{tabular}

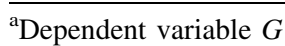

${ }^{\mathrm{b}}$ Predictors: (Constant), GC, $I, E$ 
Table 6 Summary of mediation analysis

\begin{tabular}{lcclllll}
\hline & Effect & $\mathrm{t}$ & $\mathrm{p}$ & & Effect & BootLLCI & BootULCI \\
\hline $\mathrm{GC}=>\mathrm{G}$ & 0.2 & 2.1899 & 0.0297 & $\mathrm{GC}=>E=>G$ & 0.1436 & 0.0485 & 0.2564 \\
$I=>G$ & 0.2013 & 2.2723 & 0.0242 & $I=>E=>G$ & 0.1325 & 0.0453 & 0.2442 \\
$S=>G$ & -0.0899 & -1.3371 & 0.1828 & $S=>E=>G$ & 0.1738 & 0.0945 & 0.2591 \\
\hline
\end{tabular}

\section{Discussion}

The first objective of the study was to analyse how the dimensions of Positive Mental Health vary across gender. In the current sample, females were found to have higher scores than males across variables. However, the only dimension with a significant difference in the mean scores was found to be spirituality. Thus, females are likely to be more spiritually inclined than men. This is similar to the findings of Heelas and Woodhead (2005). In their study also, it was found that females were more spiritual than males.

While researchers have suggested that spirituality and religion be treated as distinct constructs (Schlehofer et al., 2008), the Positive Mental Health Instrument includes items on Religion in the dimension of Spirituality (Vaigankar et al., 2011). The statements under spirituality referred to belief in God and the inclination to pray. A statement wise analysis revealed that on all items, females were likely to mark themselves higher except for the statement "My religious beliefs influence the way I live". On this item, males were found to have a higher mean score than females. In other words, males were more likely to allow their Religious beliefs to impact the way they lived.

The findings also suggest that there does not exist a significant correlation between Spirituality and Global affect. The direct impact was also found to be insignificant. However, the indirect path, mediated by Emotional Support was found to be significant. This implies that the level of spirituality would improve emotional support and thereby improve global affect. Thus, Spiritual inclinations are likely to result in Positive Mental Health by improving the level of Emotional Support that one can expect to receive. This finding is particularly significant given that the impact of Spirituality on Positive Mental Health has been under-explored (Vaigankar et al., 2011).

The second objective was to analyse the variance of the dimensions across Age groups. For the current study, the researchers restricted the categories to Generation $Y$ and Generation $Z$. While the mean values for Generation $Y$ were higher than those of Generation $Z$, across variables, the only significant difference was between the mean values of Interpersonal Skills. Item wise analysis found significant differences in four items; "I am willing to Compromise with people", "I try to be patient with others", "I have no trouble keeping friends" and "I am willing to share my time with others". One of the earliest studies to analyse the relationship between age and interpersonal skills was conducted by Rosen and Jerdee (1976). Their study found no significant relationship between age and interpersonal skills. A more recent study by Murry and Isaacowitz (2018) also found that age was not a significant predictor of Interpersonal skills perception.

Interpersonal Skills was also found to be significantly correlated to Global Affect. However, the indirect path was also found to be significant, suggesting that Emotional Support partially mediated the relationship between Interpersonal Skills and Global Affect. This would suggest that the better an individual's interpersonal skills, the more the
Fig. 1 Proposed model for positive mental health

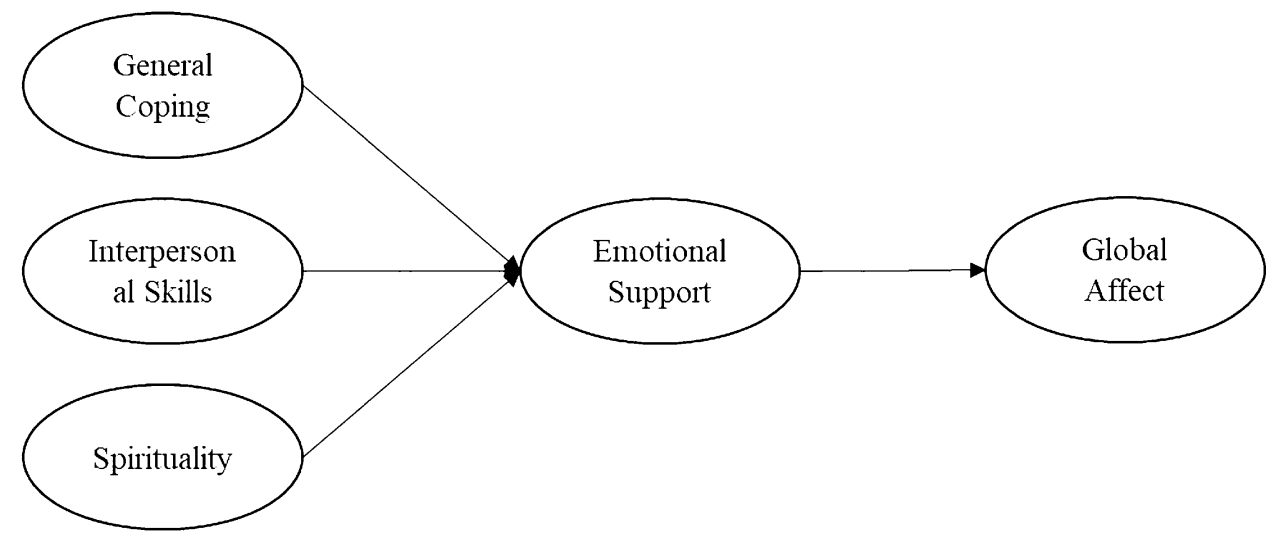


expected emotional support and more the positivity. Thus, the quality or way in which an individual handled their interpersonal relations was found to be critical for creating a support network. The findings support the results of a study by Jones et al. (2019). They found that mindfulness (describing, observing, non-judging, aware acting, non-reacting) influenced the ability to interact with empathy and promote active listening to create an emotional support structure.

The findings also suggest that General Coping was not found to be dependent on Gender or Age. A study by Skinner and Zimmer-Gembeck (2016) found that coping strategies changed with age but there were specific transition periods when these changes occurred. For example, among children, ages 5-7 and 10-12 were identified as transition points. In the current sample, Generation $Y$ was found to have a higher mean value on the dimension, however, this difference was not found to be significant suggesting that the difference would probably not hold true out of sample. The same was seen in the case of gender with females rating themselves higher on the dimension than men, however, again the difference was not found to be significant. In a similar study on the Indian population, Madalaimuthu and Kadhiravan (2017) found that the difference in coping skills of males and females was not significant. However, Lebares et al. (2018) analysed the relationship between gender and coping among physicians and found that females were more capable of coping with stressful situations.

It was also found that there is a significant relationship between General Coping and Global affect. The relationship was also found to be partially mediated by the level of emotional support. Madalaimuthu and Kadhiravan (2017) also found that General Coping and Global Affect were significantly related in the Indian Civil Service aspirants. The indirect relationship suggests that the stronger the Coping capability, more the expected emotional support and greater the positivity. This may be explained on the basis that feelings of helplessness or dependency may create feelings of depression that can cause individuals to push people away (Smith et al., 1994).

The final proposed model suggests that general coping, spirituality and interpersonal skills would help improve the perceived emotional support, which would result in positive Global Affect. In other words, the individual characteristics contributed to the ability to create a strong support network that would help in remaining positive during a crisis.

\section{Limitations and Scope for Future Research}

The biggest limitation of the current study is that it relies on perception data. Perception data would require data to be collected at different points of time to be able to establish causal relationships. However, given that the focus of the study was to understand the mental health of individuals during the pandemic, the researchers had to rely on a single point dataset. Also, the sample size of the current study is not sufficient to establish the relationships.

Additionally, since the researchers were not able to physically contact the respondents, the study relies on the ability of the respondents to comprehend the meanings of the statements and respond to the items keeping in mind the specific context of the study. Also, while all care was taken to ensure that the respondents have filled in the responses themselves and that each data set is unique, it is not possible to guarantee the same.

\section{Conclusion}

The pandemic has taken the global population by surprise. According to the WHO, COVID-19 is a new challenge and is having a devastating effect globally, its emergence and spread has caused confusion, anxiety and fear. The spread of fake news and myths has caused stigma, discrimination and fear. While the outbreak of pandemics has been known to lead to an onset of mental symptoms among the young or old. While organizations are trying to provide the required support, there is a need to understand the dimensions of positive mental health and the relationship between the dimensions.

The current study analyzed data collected from members of Generation Y and Generation Z, in India. The analysis of the collected data suggests that Global affect did not vary significantly with age or gender. The crisis appears to be impacting the positive mental health of members of both generations and gender in a similar manner. Certain individual characteristics like the General Coping Skills, Interpersonal Skills and Spiritual inclinations can impact the ability to create an Emotional Support system to guide and support one through a crisis or unstable situation and the presence of emotional support was found to be critical in remaining positive.

Authors Contributions We confirm that the manuscript has been read and approved by all named authors and that there are no other persons who satisfied the criteria for authorship but are not listed. We further confirm that the order of authors listed in the manuscript has been approved by all of us. 
Funding We wish to confirm there has been no significant financial support for this work that could have influenced its outcome.

Data Availability Data can be made available as and when required. The data have not been submitted as it contains sensitive information.

\section{Declarations}

Conflict of interest We wish to confirm that there are no known conflicts of interest associated with this publication.

\section{References}

Coronavirus Disease (COVID-19) - events as they happen. (2020). Who.int. Retrieved June 4, 2020, from https://www.who. int/emergencies/diseases/novel-coronavirus-2019/events-as-theyhappen

Demography - Working age population - OECD Data. (2020). Retrieved March 11, 2020, from https://data.oecd.org/pop/ working-age-population.htm\#: :text=The $\% 20$ working $\% 20$ age $\% 20$ population $\% 20$ is $\% 20$ defined $\% 20$ as $\% 20$ those $\% 20$ aged $\%$ 2015\%20to\%2064.,-This\%20indicator\%20measures

Ellin, A. (2014). The beat (up) generation. Psychology Today, 47(2), $56-62$.

Elmer, T., Mepham, K., \& Stadtfeld, C. (2020). Students under lockdown: Assessing change in students' social networks and mental health during the COVID-19 crisis.

Folkman, S., \& Moskowitz, J. T. (2007). Positive affect and meaningfocused coping during significant psychological stress. The scope of social psychology: Theory and applications, 193-208.

Fredrickson, B. L. (2004). The broaden-and-build theory of positive emotions. Philosophical Transactions of the Royal Society of London. Series B: Biological Sciences, 359(1449), 1367-1377.

Ganga, N. S., \& Kutty, V. R. (2013). Influence of religion, religiosity and spirituality on positive mental health of young people. Mental Health, Religion \& Culture, 16(4), 435-443.

Green, S. B. (1991). How many subjects does it take to do a regression analysis? Multivariate Behavioral Research, 26(3), 499-510.

Heelas, P., \& Woodhead, L. (2005). The spiritual revolution: Why religion is giving way to spirituality. Blackwell Pub.

Hicks, J. A., \& King, L. A. (2007). Meaning in life and seeing the big picture: Positive affect and global focus. Cognition and Emotion, 21(7), 1577-1584

Jones, S. M., Bodie, G. D., \& Hughes, S. D. (2019). The impact of mindfulness on empathy, active listening, and perceived provisions of emotional support. Communication Research, 46(6), $838-865$.

Kazmi, S. S. H., Hasan, K., Talib, S., \& Saxena, S. (2020). COVID-19 and lockdown: A study on the impact on mental health. Available at SSRN 3577515.

Lebares, C. C., Braun, H. J., Guvva, E. V., Epel, E. S., \& Hecht, F. M. (2018). Burnout and gender in surgical training: A call to reevaluate coping and dysfunction. The American Journal of Surgery, 216(4), 800-804.

Leskauskas, D. (2020). Generation Z-everyday (living with an) auxiliary ego. International Forum of Psychoanalysis (pp. 1-6). Routledge.

Madalaimuthu, A., \& Kadhiravan, S. (2017). Coping skills and positive mental health among civil service aspirants. International Journal of Education and Management Studies, 7(4), 456-459.

Marino, C., Hirst, C. M., Murray, C., Vieno, A., \& Spada, M. M. (2018). Positive mental health as a predictor of problematic internet and Facebook use in adolescents and young adults. Journal of Happiness Studies, 19(7), 2009-2022.

Murry, M. W., \& Isaacowitz, D. M. (2018). Age similarities in interpersonal perception and conversation ability. Journal of Nonverbal Behavior, 42(1), 101-111.

Nakamura, J., \& Csikszentmihalyi, M. (2009). Flow theory and research. Handbook of positive psychology, 195-206.

O'Connor, M., Sanson, A. V., Toumbourou, J. W., Norrish, J., \& Olsson, C. A. (2017). Does positive mental health in adolescence longitudinally predict healthy transitions in young adulthood? Journal of Happiness Studies, 18(1), 177-198.

Peshave, J., \& Peshave, M. (2020). Covid-19 lockdown-A blessing or curse. CLIO an Annual Interdisciplinary Journal of History, $6(1), 537-544$.

Rosen, B., \& Jerdee, T. H. (1976). The influence of age stereotypes on managerial decisions. Journal of Applied Psychology, 61(4), 428.

Schlehofer, M. M., Omoto, A. M., \& Adelman, J. R. (2008). How do "religion" and "spirituality" differ? Lay definitions among older adults. Journal for the Scientific Study of Religion, 47(3), $411-425$

Seligman, M. E. (2011). Flourish: A visionary new understanding of happiness and well-Being. NY Free Press.

Sheldon, K. M. (2012). The self-determination theory perspective on positive mental health across cultures. World Psychiatry, 11(2), 101.

Slavin, S. J., Schindler, D., Chibnall, J. T., Fendell, G., \& Shoss, M. (2012). PERMA: A model for institutional leadership and culture change. Academic Medicine, 87(1), 1481.

Smith, T. W., Christensen, A. J., Peck, J. R., \& Ward, J. R. (1994). Cognitive distortion, helplessness, and depressed mood in rheumatoid arthritis: A four-year longitudinal analysis. Health Psychology, 13(3), 213.

Spitzberg, B. H., \& Cupach, W. R. (2011). Interpersonal skills. The SAGE handbook of interpersonal communication, 481-524.

Urbina-Garcia, A. (2020). Young children's mental health: Impact of social isolation during the COVID-19 lockdown and effective strategies.

Vaingankar, J. A., Subramaniam, M., Chong, S. A., Abdin, E., Edelen, M. O., Picco, L., Lim, Y. W., Phua, M. Y., Chua, B. Y., Tee, J. Y., \& Sherbourne, C. (2011). The positive mental health instrument: Development and validation of a culturally relevant scale in a multi-ethnic Asian population. Health and Quality of Life Outcomes, 9(1), 92.

Wang, C., Pan, R., Wan, X., Tan, Y., Xu, L., McIntyre, R. S., Choo, F. N., Tran, B., Ho, R., Sharma, V. K., \& Ho, C. (2020). A longitudinal study on the mental health of general population during the COVID-19 epidemic in China. Brain, Behavior, and Immunity, 87, 40-48.

Westerhof, G. J., \& Keyes, C. L. (2010). Mental illness and mental health: The two continua model across the lifespan. Journal of Adult Development, 17(2), 110-119.

Zhang, Y., \& Ma, Z. F. (2020). Impact of the COVID-19 pandemic on mental health and quality of life among local residents in Liaoning Province, China: A cross-sectional study. International Journal of Environmental Research and Public Health, 17(7), 2381.

Zimmer-Gembeck, M. J., \& Skinner, E. A. (2016). The development of coping: Implications for psychopathology and resilience. Developmental psychopathology, 1-61.

Publisher's Note Springer Nature remains neutral with regard to jurisdictional claims in published maps and institutional affiliations. 